\title{
Modern Esophageal Function Testing and Gastroesophageal Reflux Disease in Morbidly Obese Patients
}

\author{
Ivan Kristo ${ }^{1} \cdot$ Matthias Paireder ${ }^{1} \cdot$ Gerd Jomrich $^{1} \cdot$ Daniel M. Felsenreich ${ }^{2} \cdot$ Milena Nikolic $^{1} \cdot$ Felix B. Langer $^{2}$. \\ Gerhard Prager $^{2} \cdot$ Sebastian F. Schoppmann ${ }^{1}$ iD
}

Published online: 14 June 2019

(C) The Author(s) 2019

\begin{abstract}
Background The implementation of high-resolution manometry (HRM) and the Lyon Consensus statement facilitate evaluation of gastroesophageal reflux disease and motility disorders in morbidly obese patients. Therefore, we aimed to investigate prevalence and phenotype of (borderline) GERD and esophageal motility disorders in this population.

Methods Consecutive morbidly obese $\left(B M I \geq 35 \mathrm{~kg} / \mathrm{m}^{2}\right)$ patients were offered evaluation by means of HRM, ambulatory $24-\mathrm{h}$ $\mathrm{pH}$ impedance monitoring, endoscopy, and a clinical examination at our tertiary academic center. Data were collected prospectively.

Results Out of 448 eligible individuals, 147 patients (females $=75,51 \%$ ) with a median age of 41.6 (33.4; 52.3) years and a BMI of $44(40.9 ; 49.4) \mathrm{kg} / \mathrm{m}^{2}$ were included during the study period. The Chicago Classification revealed motility disorders in 50 $(34 \%)$ patients, dominated by outflow obstruction $(18.4 \%, n=27)$ and a novel disorder $(7.5 \%, n=11)$, nicknamed jackhammer esophagus (JE). According to the Lyon Consensus, 52 (35.4\%) patients had evidence of true GERD, whereas borderline GERD was noted in another $60(40.8 \%)$. Hypersensitive esophagus was observed in $6.8 \%(n=10)$. Sensitivity and specificity of symptoms for GERD were $53.8 \%$ and $68.4 \%$, respectively.

Conclusions The current gold standard of assessment revealed that the prevalence of esophageal motility disorders and (borderline) GERD is high in the morbidly obese population. Further longitudinal data are needed to delineate the natural course of novel motility disorders like JE in obesity and to identify risk factors for adverse outcomes following bariatric surgery.
\end{abstract}

Keywords Gastroesophageal reflux disease $\cdot$ Motility disorders $\cdot$ Morbidly obese

\section{Introduction}

Due to a dramatic increase within the last decades, overweight and obesity represent a global disease burden estimated to cause 3.4 million deaths per year [1]. The relationship between obesity and deteriorating health status is already well established and bases among other factors on rising prevalence of type 2 diabetes and metabolic syndrome in obese
Sebastian F. Schoppmann

sebastian.schoppmann@meduniwien.ac.at

Ivan Kristo

ivan.kristo@meduniwien.ac.at

Matthias Paireder

matthias.paireder@meduniwien.ac.at

Gerd Jomrich

gerd.jomrich@meduniwien.ac.at

Daniel M. Felsenreich

moritz.felsenreich@meduniwien.ac.at

Milena Nikolic

milena.nikolic@meduniwien.ac.at
Felix B. Langer

felix.langer@meduniwien.ac.at

Gerhard Prager

gerhard.prager@meduniwien.ac.at

1 Department of Surgery, Upper GI Research and Service, Comprehensive Cancer Center, Gastroesophageal Tumor Unit, Medical University of Vienna, Währinger Gürtel 18-20, 1090 Vienna, Austria

2 Department of Surgery, Metabolic \& Bariatric Surgery, Medical University of Vienna, Vienna, Austria 
patients [2]. Importantly, bariatric surgery is regarded to be the best long-term solution in terms of long-term weight loss and remission of type 2 diabetes, when compared with conservative strategies [3]. Currently, sleeve gastrectomy ( $\mathrm{SG}$ ) is the leading bariatric procedure in the USA and western world [4]. Recently presented long-term data show a high incidence of hiatal hernia, gastroesophageal reflux disease (GERD), and Barrett's esophagus in patients after SG [5]. As a consequence, some authors even suggest that preexisting GERD should be a relative contraindication to SG. Furthermore, long-term data of one anastomosis gastric bypass and its consequences on biliary reflux due to a preexisting defect antireflux mechanism are still impatiently awaited.

Importantly, data on preoperative GERD status in patients undergoing bariatric surgery are more than scarce and offer only a low evidence level. Limitations include small sample sizes, selection of only symptomatic or asymptomatic patients prior to surgery and diagnosis of GERD based only on symptom perception, or even lacking cornerstones of objective testing [6-9]. Moreover, modern diagnosis of true GERD was recently redefined offering a broad spectrum of tools from symptom assessment, endoscopy, and ambulatory $\mathrm{pH}$ monitoring to esophageal function testing (EFT) ( [10]. EFT may reveal esophageal dysmotility, rather frequently observed after bariatric surgery, that potentially mimics or interferes with GERD [11, 12]. Concordantly, EFT has also been revolutionized with the development of high-resolution manometry (HRM), a fundamental technological advance in modern medicine. Moreover, implementation of novel metrics and their widespread clinical use led to an algorithm classifying esophageal dysmotility, namely, the Chicago Classification (CC) [13].

Therefore, this study was designed to rigorously assess prevalence and characteristics of esophageal motility disorders and (borderline) GERD in morbidly obese patients planned for primary bariatric surgery.

\section{Material and Methods}

Between April 2014 and December 2016, consecutive morbidly obese (BMI $\geq 35 \mathrm{~kg} / \mathrm{m}^{2}$ ) patients were offered to undergo HRM and ambulatory 24-h pH impedance monitoring at our tertiary academic center. Medical history and symptoms were assessed in a face-to-face interview with study participants being off proton pump inhibitor (PPI) for at least 10 days. In addition, esophagogastroduodenoscopy (EGD) was performed as well off PPI to determine the presence of hiatal hernia, Barrett's esophagus, and GERD, classified according to the Los Angeles (LA) classification [14]. All patients underwent informed consent, and the institutional review board approved the study protocol. Data were collected prospectively.

\section{High-Resolution Impedance Manometry}

High-resolution impedance manometry was performed using a solid-state catheter with 32 circumferential pressure transducers at $1-\mathrm{cm}$ intervals and dual impedance sensors (Sandhill Scientific, Highlands Ranch, CO, USA) as previously described [15]. Esophageal body motility was assessed with 10 liquid swallows of $5 \mathrm{ml}$ at 30 -s intervals. BioView ${ }^{\mathrm{TM}}$ (Sandhill Scientific, Highlands Ranch, CO, USA) was utilized to interpret data according to the CC v3.0 [13]. A total length below $2 \mathrm{~cm}$ and/or an intraabdominal length lower than $1 \mathrm{~cm}$ defined a structurally defective lower esophageal sphincter (LES). The LES was considered hypotensive below $10 \mathrm{mmHg}$ and hypertensive when exceeding $45 \mathrm{mmHg}$.

\section{Ambulatory 24-h pH Impedance Monitoring}

Patients had to stop PPI or H2 blocker intake at least 10 days before monitoring. A catheter containing impedance tracers and electrodes with internal reference for $\mathrm{pH}$ measuring was utilized (ComforTec ZAN-44; Sandhill Scientific, Highlands Ranch, CO, USA). Electrodes were positioned after locating the LES by HRM as previously described. Patients were instructed to stick to their daily routine and enter symptoms, body position, and meals. GERD was defined following the Lyon Consensus statement as the percentage of endoluminal $\mathrm{pH}<4$ exceeding $6 \%$ in the distal esophagus and/or endoscopic visible lesion grade $\mathrm{C}$ or $\mathrm{D}$ according to the LA classification [10]. Diagnosis of borderline GERD included patients with a total acid exposure time between 4 and $6 \%$, presence of grade A and B esophagitis, and reflux episodes exceeding 40 within $24 \mathrm{~h}$. An acid-hypersensitive esophagus was registered when normal esophageal acid exposure was associated with a symptomatic index greater than $50 \%$ and/or a symptom association probability (SAP) greater than $95 \%$. Complete bolus transit was defined as described by Tutuian et al. with complete bolus rate (CBR) being the percentage of complete bolus transit referred to all liquid swallows [16].

\section{Statistical Analysis}

Demographics are presented as mean with standard deviation if normally distributed or as median with interquartile ranges otherwise. Categorical variables are displayed as absolute numbers and percentages. HRM metrics are delineated as median with interquartile ranges. The chisquare or Wilcoxon rank test was applied as appropriate for comparison between groups. $p$ values $\leq 0.05$ were considered statistically significant. Analyses were done using 
SPSS for Macintosh Version 24.0 (IBM Corp., Armonk, NY, USA).

\section{Results}

\section{Patient Characteristics}

Out of 448 eligible individuals, 147 patients (females $=75$, $51 \%)$ with a median age of $41.6(33.4 ; 52.3)$ years and a BMI of $44(40.9 ; 49.4) \mathrm{kg} / \mathrm{m}^{2}$ were included during the study period. PPI intake was noted in $32.7 \%(n=48)$, whereas hiatal hernia was observed in $35.4 \%(n=52)$ during endoscopy. Further baseline data are outlined in Table 1.

\section{Modern Esophageal Function Testing}

The algorithm of the CCv3.0 revealed a motility disorder in 50 (34\%) patients. Outflow obstruction (OO) was noted in $18.4 \%$ $(n=27)$, JE in $7.5 \%(n=11)$, distal esophageal spasm (DES) in $4.1 \%(n=6)$, ineffective esophageal motility (IEM) in $2 \%$ $(n=3)$, and fragmented peristalsis in $1.4 \%(n=2)$ of participants. The presence of any symptoms was observed in $36.4 \%$ $(n=4)$ of individuals with JE, in $33.3 \%(n=1)$ with IEM, in $50 \%(n=1)$ with fragmented peristalsis, in $50 \%(n=3)$ with DES, and $51.9 \%(n=14)$ with OO, respectively. None of the patients diagnosed with JE had dysphagia.

\section{Lower Esophageal Sphincter}

LES assessment revealed a median total length of 4.6 (3.8; $5.4) \mathrm{cm}$ with an intraabdominal fraction of $3.4(2.3 ; 4.3) \mathrm{cm}$ and a median LES pressure of 23.8 (19; 31) $\mathrm{mmHg}$. Hypertensive and hypotensive LES were registered in 21 $(14.3 \%)$ and $10(6.8 \%)$ of patients, respectively. Structural defectiveness of the LES was observed in 13 (8.8\%) of individuals.

\section{Gastroesophageal Reflux Disease}

Symptoms were observed in $58(39.5 \%)$ of participants. According to the Lyon Consensus, 52 (35.4\%) patients had evidence of true GERD, whereas borderline GERD was noted in another $60(40.8 \%)$. Sensitivity and specificity of symptoms were $53.8 \%$ and $68.4 \%$, respectively. Criteria of the hypersensitive esophagus were met in $10(6.8 \%)$ individuals. Further characteristics of true GERD in morbidly obese patients are listed in Table 2.

\section{Differences in Phenotypes}

Patients with true GERD reported heartburn more frequently ( $p=0.0001)$ but not regurgitation and had a higher rate of
Table 1 Baseline characteristics of morbidly obese patients undergoing primary bariatric surgery

\begin{tabular}{ll}
\hline Patients & 147 \\
Sex $n(\%)$ & \\
Female & $75(51)$ \\
Male & $72(49)$ \\
Waist-to-hip ratio & $0.93(0.9 ; 1.0)$ \\
Diabetes & $47(32)$ \\
Habits $n(\%)$ & \\
Smoking & $58(39.5)$ \\
Alcohol consumption & \\
Daily & $3(2)$ \\
Frequently & $49(33.3)$ \\
Never & $95(64.6)$ \\
Daily PPI intake n $(\%)$ & $48(32.7)$ \\
Symptoms $n(\%)$ & \\
Heartburn & $49(33.3)$ \\
Regurgitation & $27(18.4)$ \\
Dysphagia & $5(3.4)$ \\
Endoscopy $n(\%)$ & \\
Hiatal hernia & $52(35.4)$ \\
Esophagitis & $46(31.3)$ \\
Barrett's esophagus & $7(4.8)$ \\
\hline
\end{tabular}

esophagitis $(p=0.0001)$, Barrett's esophagus $(p=0.043)$, and diabetes $(p=0.047)$. GERD resulted in more acidic $(p=$ 0.0001 ) episodes but did not impact on gas containing or nonacidic reflux. Borderline GERD induced more heartburn ( $p=0.013)$ but not regurgitation.

Symptoms of GERD were associated with true GERD ( $p=$ $0.008)$ and the presence of Barrett's esophagus $(p=0.011)$. There was a trend towards borderline GERD $(p=0.051)$ in asymptomatic patients. There were no differences in endoscopic or manometric findings between symptomatic or asymptomatic patients (Table 3 ).

\section{Discussion}

This is the first study evaluating a large cohort of morbidly obese patients by means of high-resolution manometry and modern gastroesophageal reflux testing. We could demonstrate that prevalence of esophageal motility disorders, classified by the Chicago algorithm, was $34 \%$, dominated by the presence of $\mathrm{OO}$ and a novel hypercontractile disorder nicknamed JE. True GERD was noted in $35.4 \%$ of participants, whereas borderline GERD was observed in another $40.8 \%$.

HRM has revolutionized modern medicine with the implementation of an hierarchical algorithm that classifies abnormality and facilitates evaluation of patients with symptoms of esophageal motility disorders and GERD [13]. Similar to 
Table 2 Phenotype of true gastroesophageal reflux disease (GERD) in morbidly obese patients

\begin{tabular}{ll}
\hline & True GERD \\
\hline Patients & 52 \\
Sex $n(\%)$ & \\
Female & $27(51.9)$ \\
Male & $25(48.1)$ \\
Age & $40.4(33.5 ; 55.2)$ \\
BMI & $43.1(40.6 ; 47.3)$ \\
Waist-to-hip ratio & $0.94(0.90 ; 1.00)$ \\
Diabetes & $22(42.3)$ \\
Symptoms $n(\%)$ & \\
Heartburn & $29(55.8)$ \\
Regurgitation & $12(23.1)$ \\
Endoscopy $n(\%)$ & \\
Hiatal hernia & $19(36.5)$ \\
Esophagitis & $28(53.8)$ \\
Barrett's esophagus & $7(13.5)$ \\
High-resolution manometry $n(\%)$ & \\
Esophageal motility disorder & $14(26.9)$ \\
Structural defective LES & $7(13.5)$ \\
Hypertensive LES & $5(9.6)$ \\
Hypotensive LES & $6(11.5)$ \\
Complete bolus rate $(\%)$ & $70(0 ; 100)$ \\
Gastroesophageal reflux disease $n(\%)$ & \\
Time pH $<4(\%)$ & $44(27 ; 60)$ \\
Total & \\
Recumbent & \\
Upright & \\
Reflux episodes & \\
Acidic & \\
Nonacidic & \\
Gas & \\
\hline & \\
&
\end{tabular}

studies using conventional manometry, HRM revealed a high rate of esophageal motility disorders in morbidly obese patients $[6,17]$. Interestingly, OO diagnosed in $18.4 \%$ of our cohort may cause chest pain and interfere with symptoms of GERD [18]. Comparatively, functional OO with intact peristalsis was observed in only $0.016 \%$ among 1000 consecutive HRM studies [19]. The association between high glucose and cholesterols levels, caused by high fat and sugar intake in morbidly obese patients, and the integrated relaxation pressure as well as an increased intraabdominal pressure, may offer an explanation for this difference in prevalence [20]. Nevertheless, therapeutic consequences and impact of bariatric surgery on the evolution of OO still remain unclear [21].

Importantly, JE, a novel hypercontractile disorder related to dysphagia and noncardiac chest pain, was observed in $7.5 \%$ of our population. Concordantly, prior trials have also observed hypercontractile disorders using conventional manometry in obesity $[6,7]$. The pathophysiological mechanism seems to include a high gastroesophageal gradient, resulting in highpressure esophageal contractions managing the functional barrier [22]. This may also be the reason for only $2 \%$ of patients diagnosed with ineffective esophageal motility in our cohort. Nevertheless, in contrast to nutcracker-like phenotypes of hypercontractility, JE is not observed in healthy controls. Moreover, longitudinal observations revealed that up to $25 \%$ of patients with JE subsequently progressed to achalasia [23]. Therefore, a therapeutic approach like peroral endoscopic myotomy is regarded as suitable treatment in these individuals [24]. Confirmatory, our group emphasized that JE is of progressive clinical nature and may even mimic GERD by responding to PPI therapy $[15,25]$. It is obvious that these facts influence bariatric strategies or even limit postoperative outcomes after surgery. Moreover, overlapping comorbidities may be misleading in identifying the underlying mechanism for symptoms after interventions. Interestingly, none of our patients with JE experienced dysphagia, whereas symptomatic response to esophageal motility disorders was also rather low. These findings go in line with observations that autonomic and sensory ganglia are susceptible to consequences of obesity like increased immune cell entry causing damage and dysregulation of sensory pathways [26].

Importantly, symptom perception is an essential alarm response to increased gastroesophageal reflux in GERD, which definitely influences the choice of surgical procedure. Interestingly, preoperative high-quality data on GERD in morbidly obese are still scarce and controversial. Fisher et al. investigated 30 morbidly obese patients and revealed that individuals with a pathologic acid exposure in the distal esophagus had a significantly higher body mass index [27]. In contrast, Lundell et al. did not observe any weight-related correlations with reflux variables in the massively obese patients [28]. So far, studies lack objective testing or diagnosis of GERD on the basis of symptoms or the presence of esophagitis, conditions that are also observed in healthy controls $[6,7]$. Recently, the Lyon Consensus proposed a modern approach for diagnosis of GERD [10]. Our data indicate that true GERD was met in $35.4 \%$, which is difficult to compare with the previous studies that diagnose GERD solely based on peptic esophagitis or symptoms [7, 17]. From a pathophysiological point of view, structural defectiveness of the LES was noted only in $8 \%$ of our cohort, whereas diabetes was significantly associated with GERD. Therefore, peripheral neuronal disorders in type 2 diabetes seem to aggravate high gastroesophageal pressure gradients and increased transient sphincter relaxation in morbid obesity and lead to the development of GERD [22, 29].

Importantly, borderline GERD was noticed in another $40.8 \%$ of our population and affected nearly half of our asymptomatic patients. Taken together, $76.2 \%$ had at least borderline evidence of GERD with $4.8 \%$ of individuals 
Table 3 Comparison of symptomatic and asymptomatic morbidly obese patients prior to bariatric surgery

\begin{tabular}{|c|c|c|c|}
\hline & Symptomatic & Asymptomatic & $p$ value \\
\hline Patients & 58 & 89 & \\
\hline $\operatorname{Sex} n(\%)$ & & & NS \\
\hline Female & $31(53.4)$ & $44(49.4)$ & \\
\hline Male & $27(46.6)$ & $45(50.6)$ & \\
\hline Age & $47.2(35.6 ; 54.9)$ & $39.2(32.8 ; 48.8)$ & 0.009 \\
\hline BMI & $44.1(40.4 ; 50.0)$ & $44(41.3 ; 49.3)$ & NS \\
\hline Waist-to-hip ratio & $0.93(0.90 ; 1.0)$ & $0.93(0.90 ; 1.02)$ & NS \\
\hline Diabetes & $19(32.8 \%)$ & $28(31.5 \%)$ & NS \\
\hline \multicolumn{4}{|l|}{ Habits $n(\%)$} \\
\hline Smoking & $26(44.8)$ & $32(36.0)$ & NS \\
\hline Alcohol consumption & & & NS \\
\hline Daily & $3(5.1)$ & 0 & \\
\hline Frequently & $20(34.5)$ & $29(32.6)$ & \\
\hline Never & $35(62.1)$ & $60(67.4)$ & \\
\hline Daily PPI intake $n(\%)$ & $34(58.6)$ & $14(16.1)$ & 0.0001 \\
\hline \multicolumn{4}{|l|}{ Symptoms $n(\%)$} \\
\hline Heartburn & $47(81.0)$ & 0 & 0.0001 \\
\hline Regurgitation & $27(46.6)$ & 0 & 0.0001 \\
\hline Dysphagia & $5(8.6)$ & 0 & 0.0001 \\
\hline \multicolumn{4}{|l|}{ Endoscopy n (\%) } \\
\hline Hiatal hernia & $24(41.4)$ & $27(30.3)$ & NS \\
\hline Esophagitis & $23(39.7)$ & $23(25.8)$ & NS \\
\hline Barrett's esophagus & $6(10.3)$ & $1(1.1)$ & 0.011 \\
\hline \multicolumn{4}{|l|}{ High-resolution manometry $n(\%)$} \\
\hline Esophageal motility disorder & $25(43.1)$ & $25(28.1)$ & NS \\
\hline Structural defective LES & $7(12.1)$ & $6(10.3)$ & NS \\
\hline Hypertensive LES & $6(10.3)$ & $15(16.9)$ & NS \\
\hline Hypotensive LES & $6(10.3)$ & $4(4.5)$ & NS \\
\hline Complete bolus rate (\%) & $45(0 ; 100)$ & $90(30 ; 100)$ & 0.021 \\
\hline \multicolumn{4}{|c|}{ Gastroesophageal reflux disease $n(\%)$} \\
\hline True GERD & $28(48.3)$ & $24(27.0)$ & 0.008 \\
\hline Borderline GERD & $18(31.0)$ & $42(47.2 \%)$ & NS \\
\hline Hypersensitive esophagus & $9(15.5)$ & 0 & 0.001 \\
\hline \multicolumn{4}{|l|}{ Time $\mathrm{pH}<4(\%)$} \\
\hline Total & $5.0(2.0 ; 9.3)$ & $3.3(1.2 ; 6.3)$ & NS \\
\hline Recumbent & $1.15(0.0 ; 6.3)$ & $0.5(0.0 ; 3.0)$ & NS \\
\hline Upright & $7.2(2.5 ; 13.2)$ & $4.65(1.9 ; 8.3)$ & 0.05 \\
\hline \multicolumn{4}{|l|}{ Reflux episodes } \\
\hline Acidic & $30(18 ; 52)$ & $30(18 ; 44)$ & NS \\
\hline Nonacidic & $14(8 ; 24)$ & $14(8 ; 20)$ & NS \\
\hline Gas & $6(3 ; 9)$ & $5(3 ; 12)$ & NS \\
\hline
\end{tabular}

$N S=$ not significant

presenting with Barrett's esophagus, which is alarming as the current data illustrate that a large percentage of de novo GERD after sleeve gastrectomy, one of the leading bariatric procedures worldwide, derives from silent preoperative reflux [30]. Based on this, adverse long-term data revealing considerable conversion rates due to GERD-related complications seem understandable [5].

Although data were collected prospectively, analysis of parameters was conducted retrospectively; thus, potential selection bias was minimized by consecutive inclusion of patients.
The number of participants and the rigorous objective evaluation according to the international gold standard represent the strength of this trial.

\section{Conclusion}

In conclusion, we were able to demonstrate that modern preoperative esophageal function and reflux testing resulted in a high rate of novel esophageal motility disorders and GERD- 
associated conditions. Further longitudinal studies are needed to delineate the course of JE in obesity and risk factors for adverse outcomes following bariatric surgery.

Acknowledgements Open access funding provided by Medical University of Vienna.

\section{Compliance with Ethical Standards}

Conflict of Interest The authors declare that they have no conflict of interest.

Ethics Statement The study was reviewed and approved by the local institutional review board according to the Helsinki Declaration or comparable ethical standards.

Statement of Informed Consent All patients underwent informed consent.

Open Access This article is distributed under the terms of the Creative Commons Attribution 4.0 International License (http:// creativecommons.org/licenses/by/4.0/), which permits unrestricted use, distribution, and reproduction in any medium, provided you give appropriate credit to the original author(s) and the source, provide a link to the Creative Commons license, and indicate if changes were made.

\section{References}

1. Ng M, Fleming T, Robinson M, et al. Global, regional, and national prevalence of overweight and obesity in children and adults during 1980-2013: a systematic analysis for the Global Burden of Disease Study 2013. Lancet. 2014;384(9945):766-81.

2. Must A, Spadano J, Coakley EH, et al. The disease burden associated with overweight and obesity. JAMA. 1999;282(16):1523-9.

3. Schauer PR, Bhatt DL, Kirwan JP, et al. Bariatric surgery versus intensive medical therapy for diabetes - 5-year outcomes. N Engl J Med. 2017;376(7):641-51.

4. Welbourn R, Hollyman M, Kinsman R, et al. Bariatric surgery worldwide: baseline demographic description and one-year outcomes from the Fourth IFSO Global Registry Report 2018. Obes Surg. 2018;28:313-22.

5. Felsenreich DM, Ladinig LM, Beckerhinn P, et al. Update: 10 years of sleeve gastrectomy-the first 103 patients. Obes Surg. 2018;28(11):3586-94.

6. Hong D, Khajanchee YS, Pereira N, et al. Manometric abnormalities and gastroesophageal reflux disease in the morbidly obese. Obes Surg. 2004;14(6):744-9.

7. Jaffin BW, Knoepflmacher P, Greenstein R. High prevalence of asymptomatic esophageal motility disorders among morbidly obese patients. Obes Surg. 1999;9(4):390-5.

8. Mathus-Vliegen LM, Tytgat GN. Twenty-four-hour $\mathrm{pH}$ measurements in morbid obesity: effects of massive overweight, weight loss and gastric distension. Eur J Gastroenterol Hepatol. 1996;8(7):635-40.

9. Korenkov M, Kohler L, Yucel N, et al. Esophageal motility and reflux symptoms before and after bariatric surgery. Obes Surg. 2002;12(1):72-6.

10. Gyawali CP, Kahrilas PJ, Savarino E, et al. Modern diagnosis of GERD: the Lyon Consensus. Gut. 2018;67(7):1351-62.

11. Borbely Y, Kroll D, Nett PC, et al. Radiologic, endoscopic, and functional patterns in patients with symptomatic gastroesophageal reflux disease after Roux-en-Y gastric bypass. Surg Obes Relat Dis. 2018;14(6):764-8.
12. Mion F, Tolone S, Garros A, et al. High-resolution impedance manometry after sleeve gastrectomy: increased intragastric pressure and reflux are frequent events. Obes Surg. 2016;26(10):2449-56.

13. Kahrilas PJ, Bredenoord AJ, Fox M, et al. The Chicago Classification of esophageal motility disorders, v3.0. Neurogastroenterol Motil. 2015;27(2):160-74.

14. Lundell LR, Dent J, Bennett JR, et al. Endoscopic assessment of oesophagitis: clinical and functional correlates and further validation of the Los Angeles classification. Gut. 1999;45(2):172-80.

15. Kristo I, Schwameis K, Paireder M, et al. Dysphagia severity is related to the amplitude of distal contractile integral in patients with jackhammer esophagus. Neurogastroenterol Motil. 2018;30(5):e13276.

16. Tutuian R, Vela MF, Shay SS, et al. Multichannel intraluminal impedance in esophageal function testing and gastroesophageal reflux monitoring. J Clin Gastroenterol. 2003;37(3):206-15.

17. Suter M, Dorta G, Giusti V, et al. Gastro-esophageal reflux and esophageal motility disorders in morbidly obese patients. Obes Surg. 2004;14(7):959-66.

18. Lin KH, Lee SC, Huang TW, et al. Esophagogastric junction outflow obstruction-related functional chest pain treated using roboticassisted thoracoscopic esophageal myotomy. J Thorac Dis. 2017;9(5):E432-E6.

19. Scherer JR, Kwiatek MA, Soper NJ, et al. Functional esophagogastric junction obstruction with intact peristalsis: a heterogeneous syndrome sometimes akin to achalasia. J Gastrointest Surg. 2009;13(12):2219-25.

20. Tanaka Y, Ihara E, Nakamura K, et al. Clinical characteristics associated with esophageal motility function. J Gastroenterol Hepatol. 2016;31(6):1133-40.

21. Ihara E, Muta K, Fukaura K, et al. Diagnosis and treatment strategy of achalasia subtypes and esophagogastric junction outflow obstruction based on high-resolution manometry. Digestion. 2017;95(1):29-35.

22. Mercer CD, Wren SF, DaCosta LR, et al. Lower esophageal sphincter pressure and gastroesophageal pressure gradients in excessively obese patients. J Med. 1987;18(3-4):135-46.

23. Huang L, Pimentel M, Rezaie A. Do jackhammer contractions lead to achalasia? A longitudinal study. Neurogastroenterol Motil. 2017;29(3)

24. Bechara R, Ikeda H, Inoue H. Peroral endoscopic myotomy for jackhammer esophagus: to cut or not to cut the lower esophageal sphincter. Endosc Int Open. 2016;4(5):E585-8.

25. Kristo I, Schwameis K, Maschke S, et al. Phenotypes of jackhammer esophagus in patients with typical symptoms of gastroesophageal reflux disease responsive to proton pump inhibitors. Sci Rep. 2018;8(1):9949.

26. O'Brien PD, Hinder LM, Callaghan BC, et al. Neurological consequences of obesity. Lancet Neurol. 2017;16(6):465-77.

27. Fisher BL, Pennathur A, Mutnick JL, et al. Obesity correlates with gastroesophageal reflux. Dig Dis Sci. 1999;44(11):2290-4.

28. Lundell L, Ruth M, Sandberg N, et al. Does massive obesity promote abnormal gastroesophageal reflux? Dig Dis Sci. 1995;40(8):1632-5.

29. Wu JC, Mui LM, Cheung CM, et al. Obesity is associated with increased transient lower esophageal sphincter relaxation. Gastroenterology. 2007;132(3):883-9.

30. Borbely Y, Schaffner E, Zimmermann L, Huguenin M, Plitzko G, Nett $P$, et al. De novo gastroesophageal reflux disease after sleeve gastrectomy: role of preoperative silent reflux. Surg Endosc. 2018.

Publisher's Note Springer Nature remains neutral with regard to jurisdictional claims in published maps and institutional affiliations. 